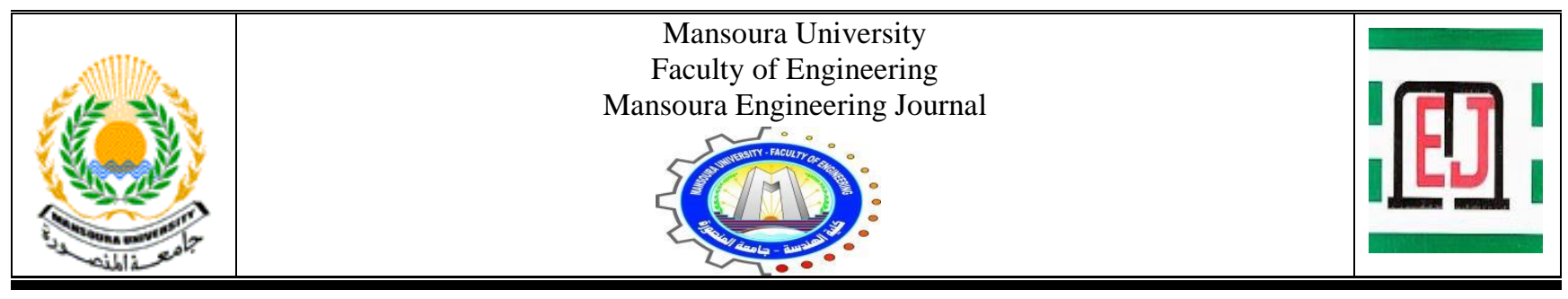

\title{
An Automated Early Detection and Classification Method for COVID-19 Stages based on deep learning technique using chest CT images
}

\author{
Mohamed Moawad Abdelsalam and Mervat El-Seddek
}

\begin{tabular}{l}
\hline KEYWORDS: \\
COVID-19; Chest CT; \\
CO-RADS Grade; Deep \\
neural network \\
\end{tabular}

\begin{abstract}
Coronavirus Disease 2019 (COVID-19) has widely spread all over the world since the ending of 2019. Until now, the death toll and injuries counted by the world's newest has not stopped. It is better to find an automatic classification technique to find out the extent of pneumonia, and be helpful tools for faster decisions in clinical practice. The Early detection of COVID-19 is an important and urgent need for stopping the spread of the disease. The aim of this study is to develop an automated early detection and classification method for COVID-19 patients based on deep learning technique using chest CT images. The proposed technique classifies the five COVID-19 infection grades with accuracy $98 \%$. It based on using a deep convolutional neural network (CNN) with the ResNet50 model. It detects the early infection grades with a precision 98.3\% . Several statistical performance measures had been evaluated for multiclassification categories. The proposed technique helps the physicians to make faster decisions and treatments for different COVID-19 grades.
\end{abstract}

\section{INTRODUCTION}

$\mathrm{S}$ INCE the Coronavirus Disease 2019 (COVID-19) was first discovered in Wuhan, China, last December 2019, the death toll and injuries counted by the world's newest has not stopped [1]. The influence of Covid-19 varies between persons. Most people with the infection experience mild to moderate symptoms that include common symptoms: fever, fatigue, and cough. Some people may have shortness of breath, aches, nasal congestion, throat pain and diarrhea [2].

Received: (30 November, 2020) - Revised: (20 March, 2021) - Accepted: (29 March, 2021)

Corresponding Author: Mohamed Moawad Abdelsalam, Assistant Professor at Computers and Systems dep., Faculty of Engineering, Mansoura University (e-mail: mohmoawed@yahoo.com ).

Mervat El-Seddek, Assistant Professor at Department of Electronics and Communications - Misr Higher Institute for Engineering and Technology MansouraUniversity (e-mail: mervat.elseddek@ieee.org).
It takes an average of 5-6 days between a person's infection with the COVID-19 and the onset of symptoms; however, it may take up to 14 days [3-4]. Older people who have existing chronic medical conditions, such as heart disease, lung disease or diabetes may be at higher risk of serious illness [5]. As of this time, there is no known specific, effective, proven, pharmacological treatment.

The COVID-19 has multiple effects on patient lungs: 1 ) Ground Glass Opacity (GGO) pattern: is the most common finding infections. They are usually multifocal, bilateral and peripheral. 2) Crazy Paving: is a combination of GGO with interlobular and intralobular thickened lines. 3) Vascular dilatation and 4) Traction Bronchiectasis [6-8].

The real-time polymerase chain reaction (RT-PCR) is the standard diagnostic method that is used to detect COVID-19 [9]. Patient samples are taken from the most infected COVID19 places like the back of the nose or mouth or deep inside the lungs. Although, the PCR test has high sensitivity and quick 
results, it may negatively diagnose the COVID-19 patients in the early stages due to the eliciting of false-negative and falsepositive results [10-14]. The early diagnosis of COVID-19 is crucial for disease treatment and control. Lung and chest CT imaging may be a more reliable and rapid method to diagnose COVID-19 especially early infections. CT imaging for COVID-19 reveals areas of (GGO) with bilateral peripheral involvement in multiple lobes progressing to "crazy-paving" patterns.

Artificial intelligence techniques especially deep neural networks have become one of the most important methods used in medical diagnose [15-17]. In [18] they developed automated CT image analysis tools to distinguish between coronavirus patients and disease-free. A deep learning-based system for chest CT images automatic segmentation [19]. COVID-19 and Influenza-A viral pneumonia were distinguished by [20] using deep learning techniques for pulmonary CT images. Another deep learning method is used to extract the COVID-19 graphical features for clinical diagnose [21].

As in [22], they introduced a Convolution Neural Network (CNN) model for COVID-19 subjects' detection using both chest CT and X-ray images dataset. Another COVID-19 detection model was introduced based on clinical features and radiological semantics [23]. Using chest X-ray images, a detection model based on the DarkNet model was proposed as a classifier [24]. In [25], they used An Adaptive Feature Selection guided Deep Forest (AFS-DF) to classify COVID19 patients based on using chest CT images. Other CNN models were used to classify different CT samples with noinfection, Influenza viral pneumonia or COVID-19 [26]. In [27] deep learning methods were used to extract the graphical features of COVID-19 for clinical diagnosis. Also, the severity of COVID-19 was predicted in [28]. They used the chest CT images with a deep learning model. The images' findings were compared with the pneumonia severity index (PSI). A Random Forest (RF) model was used in [29] for COVID-19 severity level detection.

\section{A. CO-RADS as a Reporting and Data System for COVID-19}

To find out the level of suspicion of COVID-19, the Dutch Society of Radiology proposed the CO-RADS grading system. The measuring system begins with CO-RADS 1 to CO-RADS 6. The higher the number, the higher of COVID-19 infection status. Table 1 shows the CO-RADS classification grades [12]. From Table 1, PCR diagnosing test is negative in grades CO-RADS 1 to CO-RADS 3, while it is positive detection in other grades. Therefore, the physicians cannot be count on PCR as a diagnosing tool for the early detection of the infected cases.
TABLE 1

CO-RADS CLASSIFICATION FOR COVID-19

\begin{tabular}{c||c||c||c}
$\begin{array}{c}\text { CO-RADS } \\
\text { Grade }\end{array}$ & Infection Type & CT Findings & $\begin{array}{c}\text { PCR } \\
\text { Findings }\end{array}$ \\
\hline CO-RADS 1 & No & $\begin{array}{c}\text { Normal or } \\
\text { non-infectious } \\
\text { abnormalities }\end{array}$ & Negative \\
\hline CO-RADS 2 & Low & $\begin{array}{c}\text { Abnormalities } \\
\text { consistent with } \\
\text { infections other than } \\
\text { COVID-19 }\end{array}$ & Negative \\
\hline CO-RADS 3 & Indeterminate & $\begin{array}{c}\text { Unclear whether } \\
\text { COVID-19 is } \\
\text { present }\end{array}$ & Negative \\
\hline CO-RADS 4 & High & $\begin{array}{c}\text { Abnormalities } \\
\text { suspicious for } \\
\text { COVID-19 }\end{array}$ & Positive \\
\hline CO-RADS 5 & Very High & Typical COVID-19 & Positive \\
\hline CO-RADS 6 & PCR + & & Positive
\end{tabular}

The aim of this paper is to develop an automated early detection and classification method for COVID-19 patients based on deep learning technique using chest CT images, with finding out the abnormalities locations. As well as, a classification technique to enable the differentiation of the coronavirus patients (early detection grades CO-RADS 2 and CO-RADS 3) from those who do not have the disease (CORADS 1). The proposed technique depends on using a pretrained CNN based ResNet50 model. Providing the accurate measurements to ensure the effectiveness of the developed technique.

\section{B. The study Contributions}

The main contributions of the proposed technique can be as:

- This study has used the Chest CT images, which can be obtained easily, quickly and without difficulty and not waiting for PCR test results.

- The early detection of COVID-19 in early grades is helpful in fast treatment and healthcare.

- Full classification grades from normal grade (CO-RADS 1) to highly infected grades (CO-RADS 5)

- Standalone technique and a complete end-to-end system.

- Quite a high classification performance measures, although the lower datasets, these results can be enhancement with time by increasing the training datasets.

\section{Materials}

One-hundred and fifty chest CT patient images' were retrospectively reviewed with suspected COVID-19 pneumonia from February to 5th of April 2020. The patients' data were collected from different resources as Chest hospitalMinistry of Health and Population- Mansoura- Egypt, Radiology Assistant website datasets and Rad2Share COVID19 datasets. Patients were divided into CO-RADS1 to CORADS 5 according to their clinical diagnose, laboratory and CT findings. Thirty cases have no abnormality or infections (CO-RADS 1). The remaining 120 patient datasets were divided into 30 subjects for each grade from CO-RADS 2 to CO-RADS 5. The patients had abnormal CT findings that 
suggested pneumonia as the diagnosis. Most patients suffer from cough, fever and shortness of breath symptoms.

\section{MeTHODS}

The subjects' images were processed using a developed custom MATLAB program for contrast enhancement and abnormalities detection. The classification technique is shown in Fig. 1 and summarized as follow:

- The inputs are chest CT images, which contain information about the lung infection or normality status. Firstly, the images were cropped and rescaled to be equal in size with $224 \times 224$ pixels.

- Then apply image-processing tools for contrast enhancement. The images are pre-processed through several steps to ensure good results of the deep neural network.

- The resulted enhancement images were analyzed to create the gray level matrix. Then apply image segmentation tools for calculating the image pixels weights based on grayscale intensity for abnormalities demonstration and features extraction.

- The final preparing image step is the coloring process; hence the pixels were colored according to their weights for illustrating the infected regions.

- The main problem in medical image analysis is the limited data sets, while deep learning models often need many big datasets. Transfer learning's main advantage is allowing the use of fewer datasets for training, with less calculation time and fewer costs. In this research, a deep convolutional neural network (CNN) was built based ResNet50 model for the classification of COVID-19 lung CT images. The proposed CNN hyperparameters can be as follow: 1) it consists of three types of layers with multiclassification categories. 2) The first and second layers are called convolutional layer and pooling layer respectively, each layer has 32 filters. 3) The last classification layer has two fully connected layers with 128 neurons. 4) The network-learning rate is $10^{-4}$. Over a million images from the ImageNet database were used for a pre-trained network.

The COVID-19 classification technique has two objectives. i) Classify the COVID-19 CO-RADS grades to normal (CO-RADS 1), Mild (CO-RADS 2), Moderate (CORADS 3), Severe (CORADS 4) and very high infected (CORADS 5). ii) Early detection of COVID especially in early levels enables the specialist for treatments and avoids rapid progression stage. The classification technique flowchart is shown in Fig.2. The procedure classification results can be concluded in Fig.3.

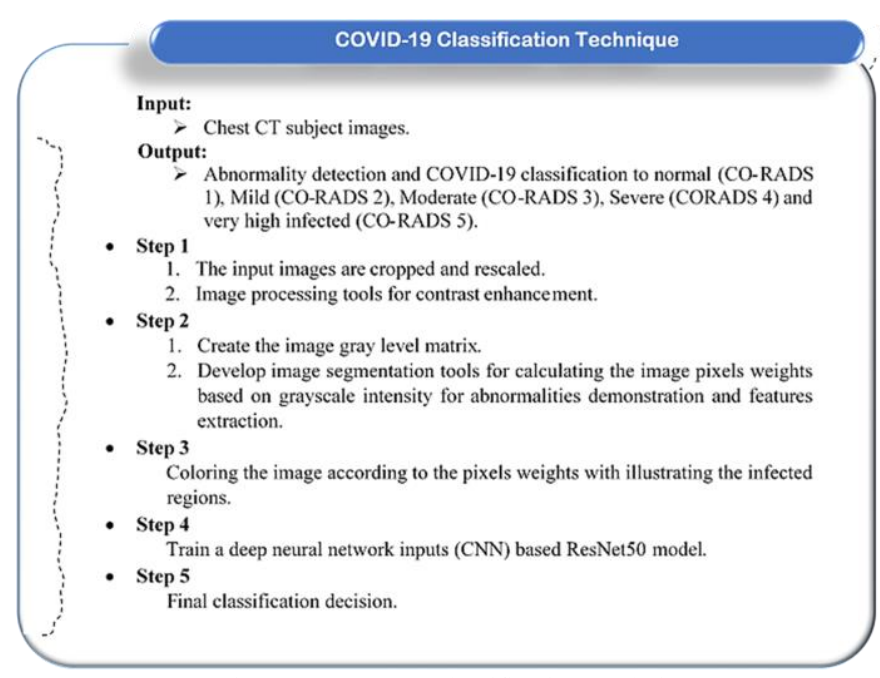

Fig.1 . COVID-19 Classification Technique

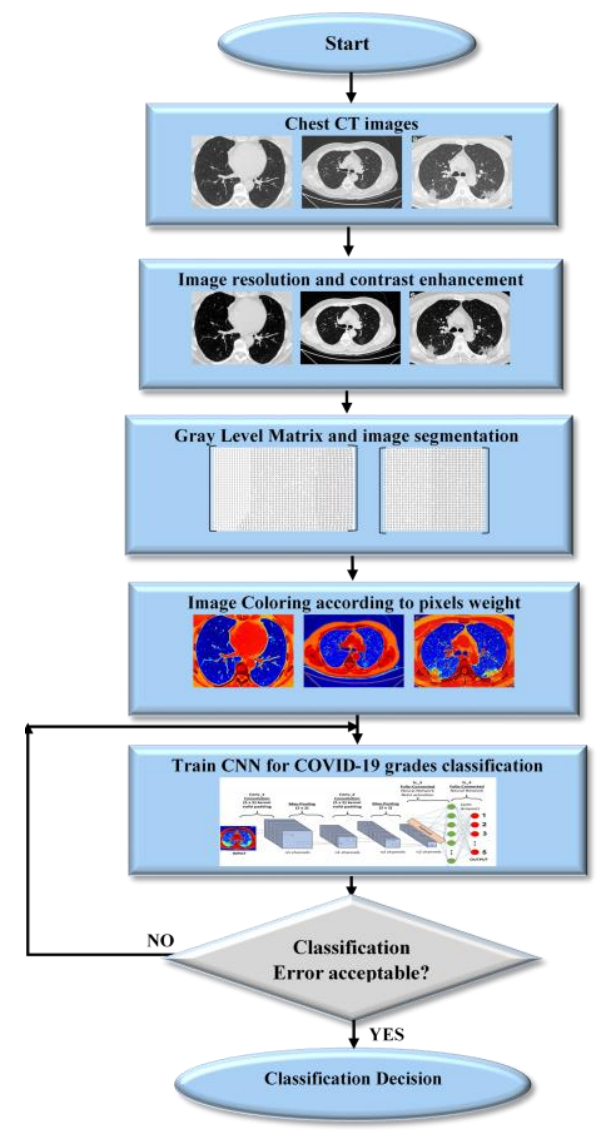

Fig.2: The proposed classification technique Flowchart

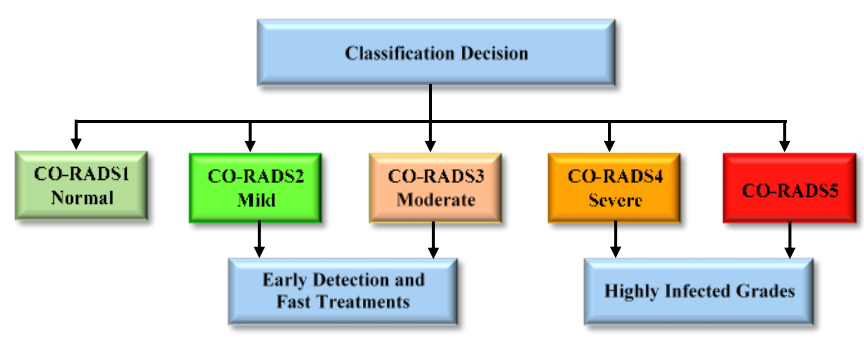

Fig.3. The Procedure Classification Results 


\section{RESUlTS AND DISCUSSION}

One hundred and fifty subjects were checked up. The RTPCR test was negative for 90 subjects and positive for 60 subjects. The subjects are categorized as 30 subjects for normal CT lungs (CO-RADS 1) and 30 subjects for each infected grades from CORADS 2 grade to CORADS 5 grade. Table 2 shows the demographic characteristics of the studied Subjects. Table 3 shows the patients' symptoms.

TABLE 2

Demographic Characteristics of the studied Subjects

\begin{tabular}{c||c||c}
\multicolumn{1}{c||}{ Grade } & $\begin{array}{c}\text { Age }(\mathbf{y}) \\
\text { Average } \pm \text { STD }\end{array}$ & $\begin{array}{c}\text { Female/Male } \\
(\mathbf{N})\end{array}$ \\
\hline CO-RADS 1 $(\mathbf{N}=30)$ & $56.7 \pm 10$ & $13 / 17$ \\
\hline CO-RADS 2 $(\mathbf{N}=30)$ & $56.37 \pm 15$ & $18 / 12$ \\
\hline CO-RADS 3 $(\mathbf{N}=30)$ & $55.9 \pm 13.13$ & $10 / 20$ \\
\hline CO-RADS 4 $(\mathbf{N}=30)$ & $62.17 \pm 14.43$ & $16 / 14$ \\
\hline CO-RADS 5 $(\mathbf{N}=30)$ & $57.9 \pm 11.72$ & $11 / 19$ \\
\hline p-value & 0.3332 & 0.1063
\end{tabular}

TABLE 3

\begin{tabular}{|c||c||c|}
\multicolumn{2}{c||}{ THE PATIENTS' SYMPTOMS } \\
\hline Symptoms & Subject No. & Percentage \\
\hline Fever & $130 / 150$ & 86.67 \\
\hline Coughing & $68 / 150$ & 45.33 \\
\hline $\begin{array}{c}\text { Shortness of } \\
\text { breath }\end{array}$ & $45 / 150$ & 30 \\
\hline Abdominal pain & $12 / 150$ & 8 \\
\hline
\end{tabular}

Table 2 illustrates the subject's distribution, mean ages with their standard deviations and sex. The $\mathrm{p}$-value was selected to be statistically significant at $\mathrm{p}<0.005$. The ages' subjects have $\mathrm{p}=0.3332(\mathrm{p}>0.05)$, and the female/male subjects were $68 / 84$ has $\mathrm{p}=0.1063$. Therefore, the demographic characteristics were statistically non-significant, i.e, neither ages nor sex affects the studied results. Table 3 summarizes the patients' Symptoms. The patients who suffer from fever are $86.67 \%$, while $45.33 \%$ patients suffer from coughing. Shortness of breath affected $30 \%$ of studied patients' samples, while abdominal pain affected $8 \%$ of them.

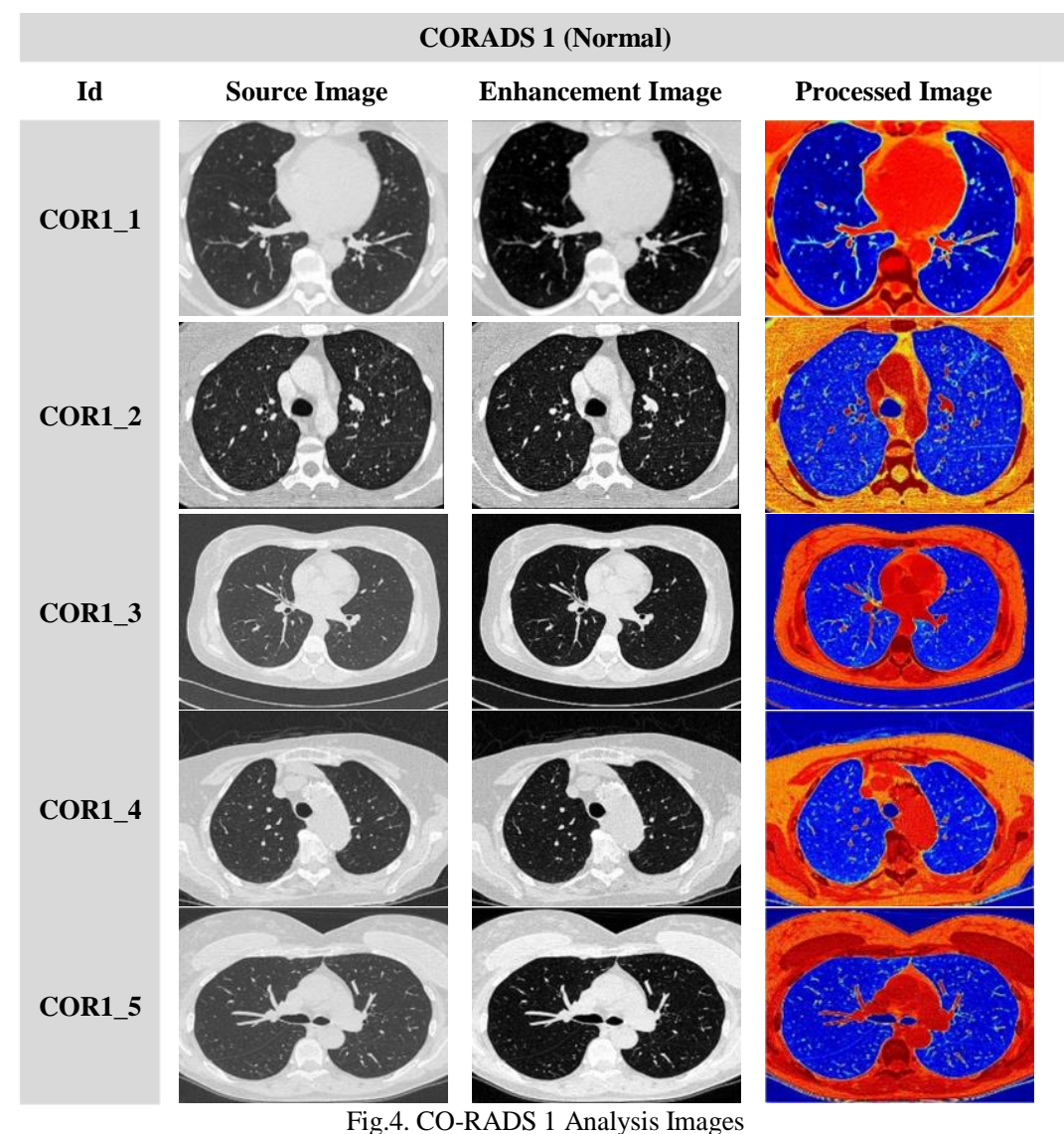




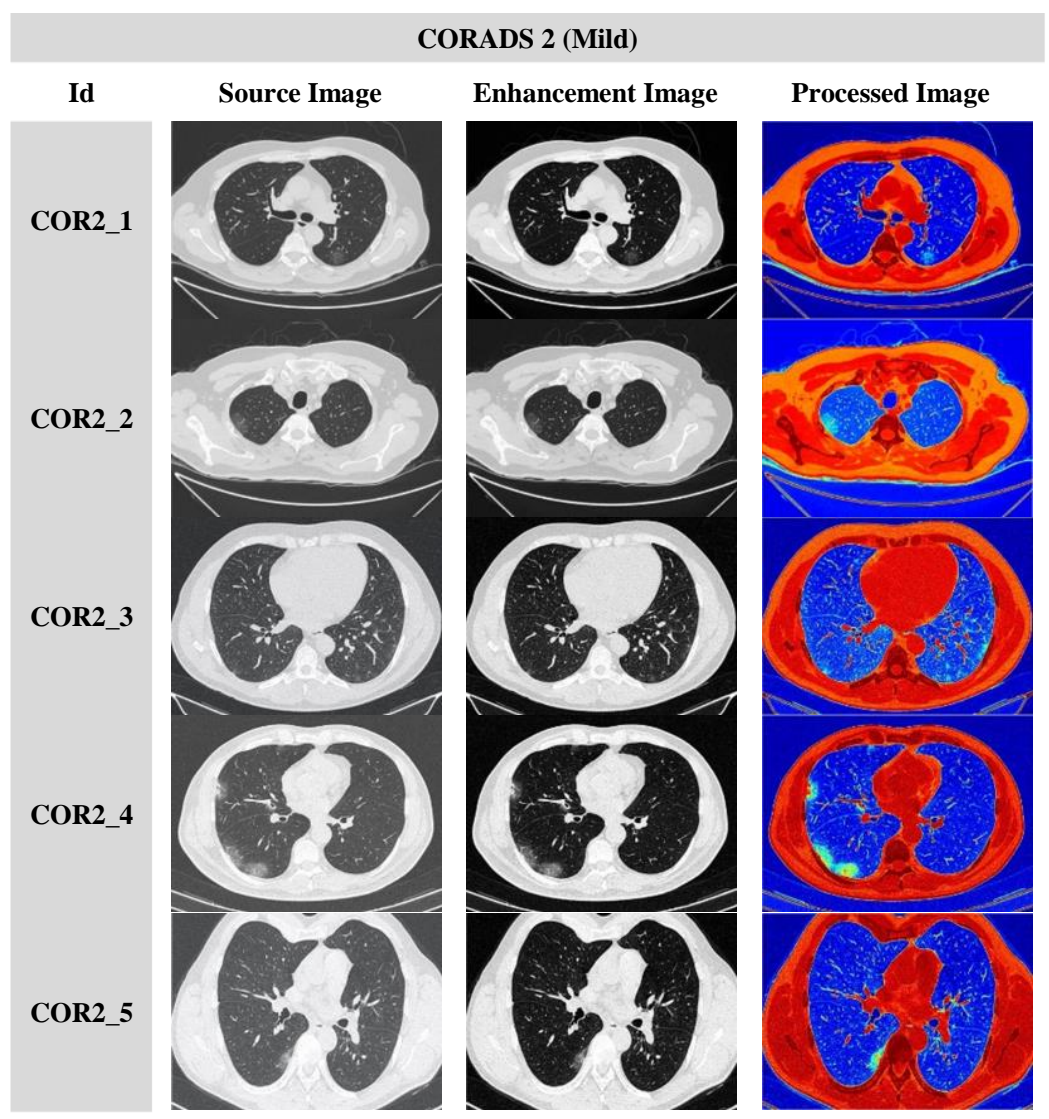

Fig.5. CO-RADS 2 Analysis Images

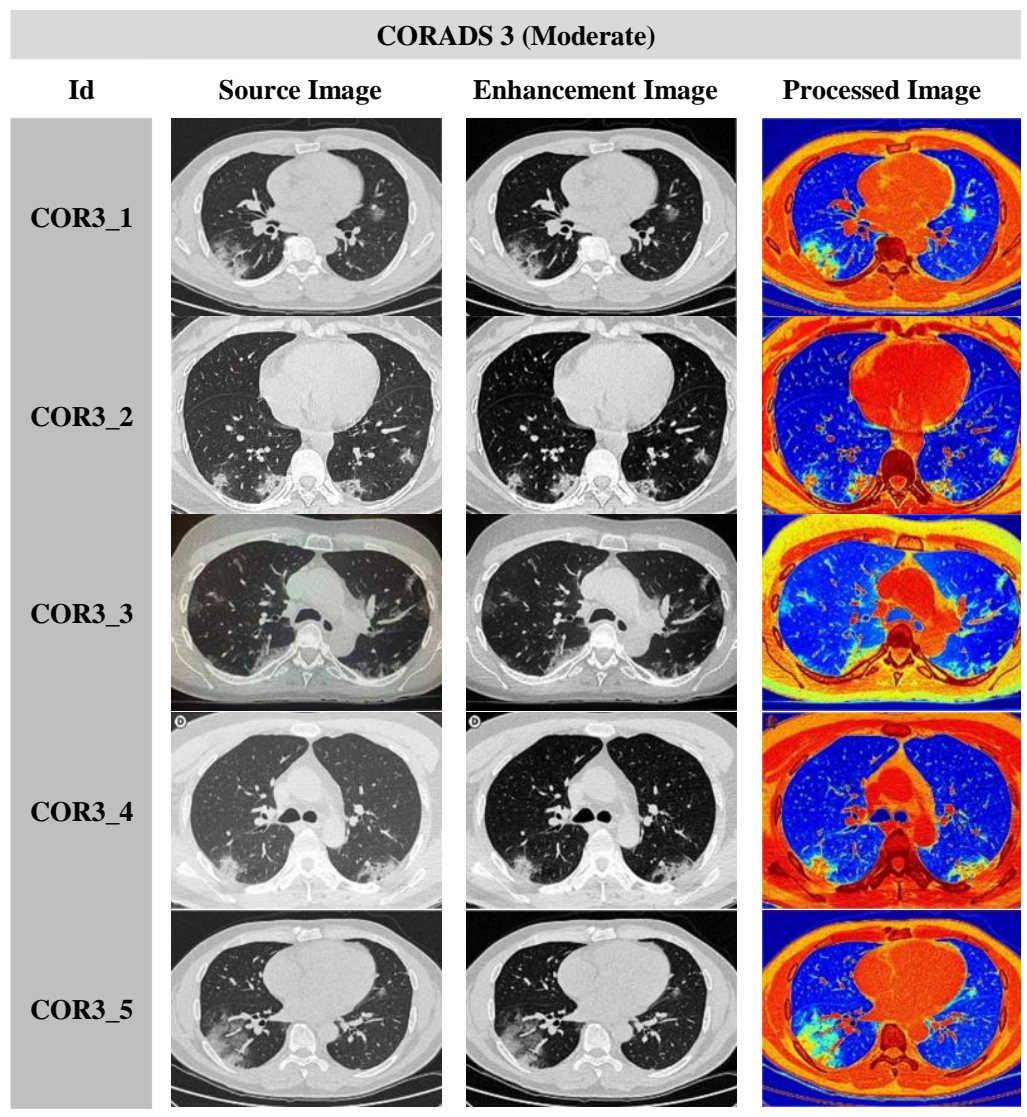

Fig.6. CO-RADS 3 Analysis Images 


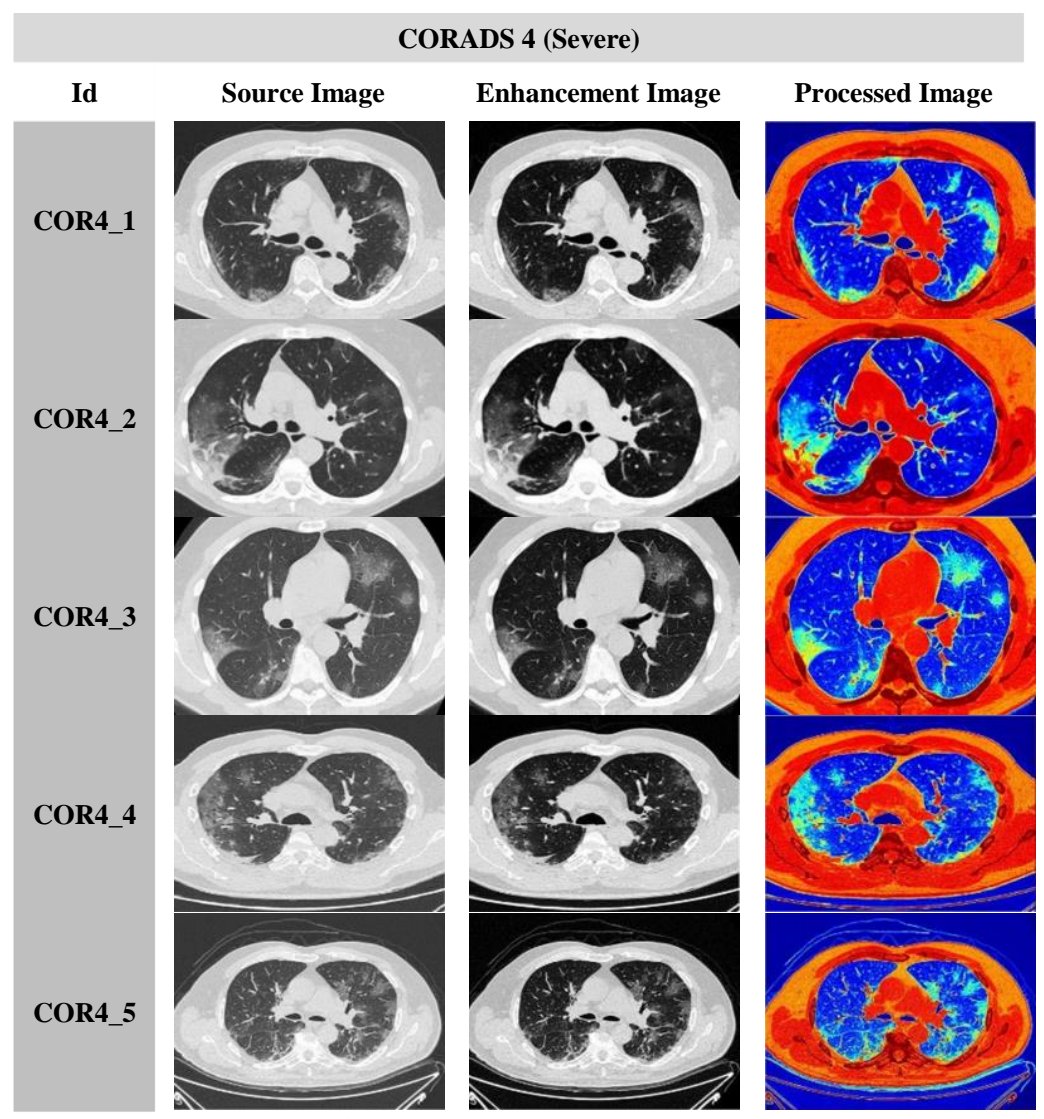

Fig.7. CO-RADS 4 Analysis Images

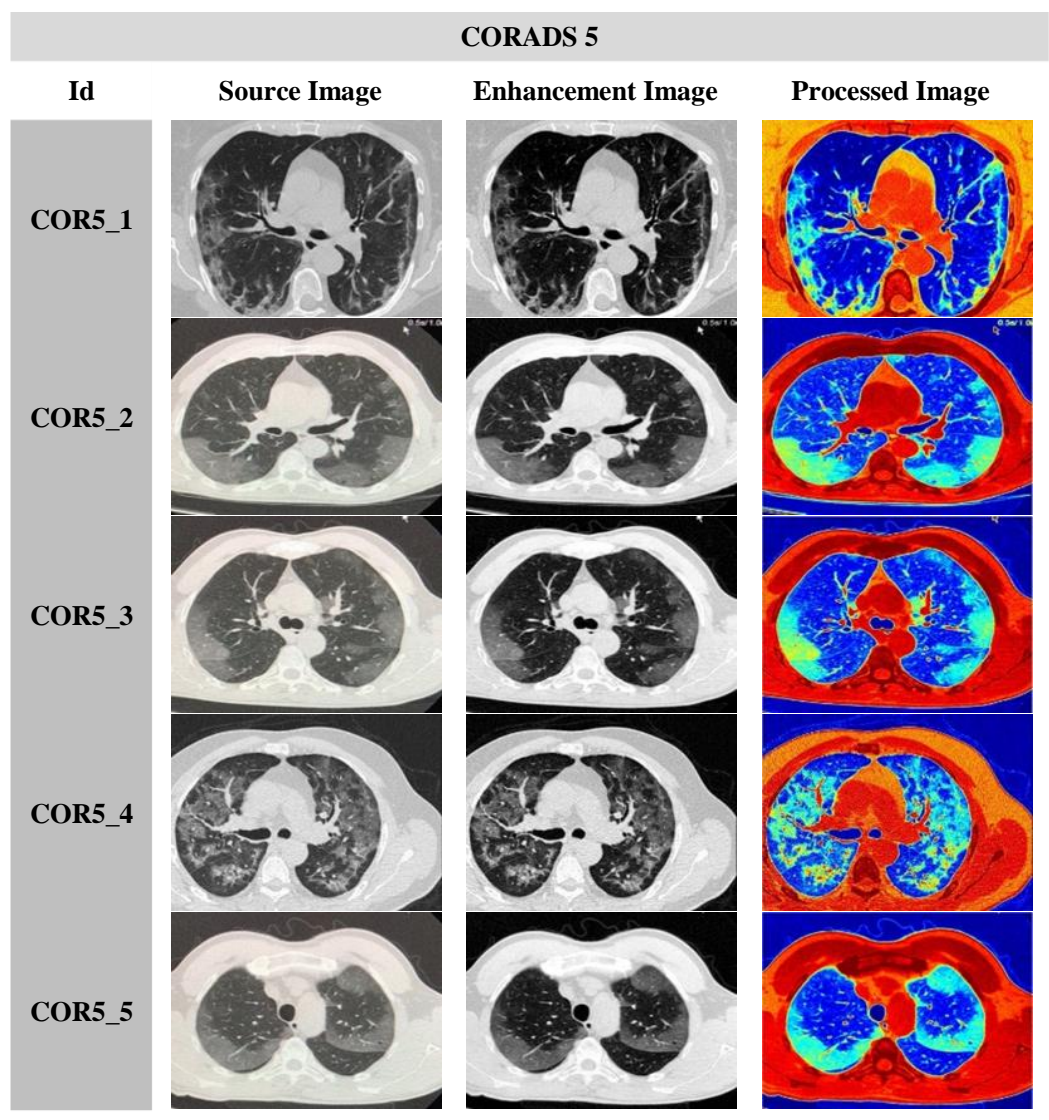

Fig.8. CO-RADS 5 Analysis Images 


\section{A. Image Analysis Results}

The Image analysis results are shown in Fig.4 to Fig.8. Figure 4 to Fig. 8 contain 5 sample processed images for each grade. The first column contains the source CT lung images, while the second column contains the images after contrast enhancement. The third column contains the colored images with abnormalities demonstration after gray matrix and image segmentation. The figures deduce that the abnormalities such as GGO and crazy paving regions are increased with high infection grades. The colored images were used as CNN inputs for training. The datasets are divided into training datasets with 125 subjects, while the testing datasets contain 25 new subjects ( 5 new subjects for each grade). The classification performances are shown in the next section.

\section{B. The Classification performances}

The classification performance measures are summarized in Table 4 to Table 8 . Table 4 shows that, the classification accuracy reaches $98 \%$. The classification technique correctly classifies 147 subjects, while it misclassifies 3 subjects only. For a full comparison, two subcategories COVID Stage1 and COVID Stage 2 were labeled. COVID Stage 1 contains the early COVID grades as CO-RADS1 and CO-RADS2. While COVID Stage 2 contains the highly infected COVID grades as CO-RADS4 and CO-RADS5.

TABLE 4

\begin{tabular}{|c|c|c|c|c|c|c|}
\hline \multicolumn{7}{|c|}{ CLASSIFICATION ACCURACY DATA } \\
\hline & Normal & \multicolumn{2}{|c|}{$\begin{array}{c}\text { COVID } \\
\text { (Stage 1) }\end{array}$} & \multicolumn{2}{|c|}{$\begin{array}{c}\text { COVID } \\
\text { (Stage 2) }\end{array}$} & \multirow[b]{2}{*}{ Total } \\
\hline CORADS Stage & CO-RADS 1 & CO-RADS 2 & CO-RADS 3 & CO-RADS 4 & CO-RADS 5 & \\
\hline Subjects (N) & 30 & 30 & 30 & 30 & 30 & 150 \\
\hline $\begin{array}{c}\text { Correctly } \\
\text { classified images }\end{array}$ & 30 & 29 & 29 & 29 & 30 & 147 \\
\hline $\begin{array}{c}\text { False classified } \\
\text { images }\end{array}$ & 0 & 1 & 1 & 1 & 0 & 3 \\
\hline \multicolumn{7}{|c|}{ Classification Accuracy } \\
\hline
\end{tabular}

Tables 5 to 8: four Confusion Matrices had been established for the classification results normal versus infected subjects, the early stage COVID (ST1) versus the highly infected stages COVID (ST2), early-infected stages and highly infected grades.

Table 9: The classification of normal and infected subjects has $100 \%$ sensitivity because of the clarity of normal images compared to the infected ones. Therefore, the classification technique correctly classifies the normal images, with $97.5 \%$ specificity and $90.9 \%$ precision. In classification, early COVID-19 grades with highly infected grades, the sensitivity, specificity and precision were $96.67 \%$, $98.33 \%$ and $98.3 \%$ respectively. While the classification results for early-infected grades are $96.67 \%$ sensitivity, $96.67 \%$ specificity and $96.67 \%$ precision. In addition, highly infected grades are $96.67 \%$ sensitivity, $100 \%$ specificity and $100 \%$ precision.

TABLE 5

CONFUSION MATRIX NORMAL VERSUS INFECTED CASES

\begin{tabular}{c||c||c} 
Actual status & Classified Normal & Classified Infected \\
\hline $\begin{array}{c}\text { Normal } \\
(30)\end{array}$ & $\begin{array}{c}30 \\
\text { TP (True Positive) }\end{array}$ & FN (False negative) \\
\hline $\begin{array}{c}\text { Infected } \\
(120)\end{array}$ & 3 & 117 \\
\hline
\end{tabular}

TABLE 6

CONFUSION MATRIX COVID (ST1) VERSUS COVID (ST2)

\begin{tabular}{c||c||c} 
Actual status & $\begin{array}{c}\text { Classified } \\
\text { COVID (ST1) }\end{array}$ & $\begin{array}{c}\text { Classified } \\
\text { COVID (ST2) }\end{array}$ \\
\cline { 1 - 2 } $\begin{array}{c}\text { COVID }(\text { ST1 }) \\
(60)\end{array}$ & $\begin{array}{c}58 \\
\text { TP (True Positive) }\end{array}$ & FN (False negative) \\
\hline $\begin{array}{c}\text { COVID }(S T 2) \\
(60)\end{array}$ & 1 & 59 \\
FP (False Positive) & TN (True Negative)
\end{tabular}

TABLE 7 CONFUSION MATRIX COVID (ST1)

\begin{tabular}{c||c||c} 
Actual status & $\begin{array}{c}\text { Classified } \\
\text { CO-RADS 2 }\end{array}$ & $\begin{array}{c}\text { Classified } \\
\text { CO-RADS 3 }\end{array}$ \\
\hline $\begin{array}{c}\text { CO-RADS 2 } \\
(30)\end{array}$ & $\begin{array}{c}29 \\
\text { TP (True Positive) }\end{array}$ & FN (False negative) \\
\hline $\begin{array}{c}C O-R A D S 3 \\
(30)\end{array}$ & 1 & 29 \\
FP (False Positive) & $\mathrm{TN}$ (True Negative)
\end{tabular}

TABLE 8. CONFUSION MATRIX COVID (ST2)

\begin{tabular}{c||c||c} 
Actual status & $\begin{array}{c}\text { Classified } \\
\text { CO-RADS 4 }\end{array}$ & $\begin{array}{c}\text { Classified } \\
\text { CO-RADS 5 }\end{array}$ \\
\hline $\begin{array}{c}\text { CO-RADS 4 } \\
(30)\end{array}$ & $\begin{array}{c}29 \\
\text { TP (True Positive) }\end{array}$ & FN (False negative) \\
\hline $\begin{array}{c}\text { CO-RADS } 5 \\
(30)\end{array}$ & 0 & 30 \\
FP (False Positive) & TN (True Negative)
\end{tabular}


TABLE 9

SENSITIVITY, SPECIFICITY AND PRECISION PARAMETERS

\begin{tabular}{|c|c|c|c|c|}
\hline $\begin{array}{l}\text { Performance } \\
\text { Parameters }\end{array}$ & $\begin{array}{l}\text { Normal } \\
\text { vs. } \\
\text { Infected }\end{array}$ & $\begin{array}{l}\text { COVID } \\
\text { (ST1) } \\
\text { vs. } \\
\text { COVID } \\
\text { (ST2) }\end{array}$ & $\begin{array}{c}\text { COVID } \\
\text { (ST1) }\end{array}$ & $\begin{array}{c}\text { COVID } \\
\text { (ST2) }\end{array}$ \\
\hline $\begin{array}{l}\text { Sensitivity (\%) } \\
\frac{\boldsymbol{T P}}{\boldsymbol{T P}+\boldsymbol{F N}} \times 100\end{array}$ & 100 & 96.67 & 96.67 & 96.67 \\
\hline $\begin{array}{l}\text { Specificity (\%) } \\
\frac{\boldsymbol{T N}}{\boldsymbol{T N}+\boldsymbol{F P}} \times \mathbf{1 0 0}\end{array}$ & 97.5 & 98.33 & 96.67 & 100 \\
\hline $\begin{array}{l}\text { Precision (\%) } \\
\frac{\boldsymbol{T P}}{\boldsymbol{T P}+\boldsymbol{F P}} \times \mathbf{1 0 0}\end{array}$ & 91 & 98.3 & 96.67 & 100 \\
\hline $\begin{array}{c}\text { Misclassification } \\
\text { Error }(\%) \\
\boldsymbol{F P}+\boldsymbol{F N} \\
\boldsymbol{P}+\boldsymbol{T N}+\boldsymbol{F P}+\boldsymbol{F N} \\
\mathbf{1 0 0}\end{array}$ & 2 & 2.5 & 3.33 & 1.67 \\
\hline
\end{tabular}

One hundred and fifty images are the main training datasets. Unfortunately, the RT-PCR test cannot detect the abnormalities in the earlier stages. Therefore, the PCR test was negative for the early 90 subjects and positive for the remaining 60 high-infected subjects. The proposed classification technique uses the convolutional neural network-based ResNet50 model. The aim of the classification technique is to classify the five grades of COVID-19 infection, as well as the early diagnosis and treatment for the early detected grades. The classification accuracy reaches $98 \%$ with the sensitivity ranges from $100 \%$ to $96.7 \%$ according to the classification test. The average specificity is $98.12 \%$ and $96.5 \%$ average precision. The minimum classification error is $1.67 \%$ and reaches its maximum of $3.33 \%$ at COVID Stage1 classification. There is a similarity between the three-misclassification images, as they express the intermediate cases of infection. These results can be easily improved by increasing the number of images used in the network training.

To evaluate the effectiveness of the proposed technique, a patient evaluation with time has been processed as shown in Fig.9. The figure shows three CT scans for coronavirus patient throughout the disease. The first CT scan image was taken on $27^{\text {th }}$ January 2020 after 4 days of COVID-19 symptoms. The processed images shows, opacities appear in the upper- and mid-lungs, with lower less opacities in the lower lobes. The second CT scan was obtained on $31^{\text {st }}$ of January 2020. Opacities have been reduced within the lung in the processed image. The third CT scan was taken on $15^{\text {th }}$ February 2020, no opacities are present.

\section{Comparative Study}

In addition to the obtained results, the effectiveness of the proposed classification technique can be evaluated by comparing it with other studies considering the same classification topic. Table 10 illustrates several COVID-19 classification techniques. Several classification objectives were listed in the table.

In [26], they used deep learning for classification between COVID-19, Influenza viral pneumonia, and noinfection with $98 \%$ accuracy, $98.2 \%$ sensitivity and $92.2 \%$ specificity. The same classification objective in [27] by using a convolutional neural network with $73.1 \%$ accuracy, $67 \%$ sensitivity and $74 \%$ specificity. The classification between COVID-19 patients and no-infection were presented in [25], they reached $91.8 \%$ accuracy, $93.1 \%$ sensitivity and $89.9 \%$ specificity. The severity of COVID-19 patients were classified in [28] and [29] with 89\% accuracy, $82.2 \%$ sensitivity and $82.8 \%$ specificity; and $87.5 \%$ accuracy, $93.3 \%$ sensitivity and $74.5 \%$ specificity respectively. The proposed technique is used for the classification of COVID-19 stages with $98 \%$ accuracy, the sensitivity lies between $96.67 \%$ and $100 \%$ according to the classification stages. As well, the specificity is between $975 \%$ and $100 \%$. From Table 10, the suggested methodology has achieved the best results, this is due to not using the source image directly or even enhance the contrast only, but depend on applying the image-processing tools for contrast enhancement through several steps to ensure good results and enhance the image gray level. Then the resulted enhancement images have been analyzed to create the gray level matrix. After that, applying the image segmentation tools for calculating the image pixels weights based on grayscale intensity for abnormalities demonstration and features extraction. These results can be enhanced with the increasing number of training datasets.

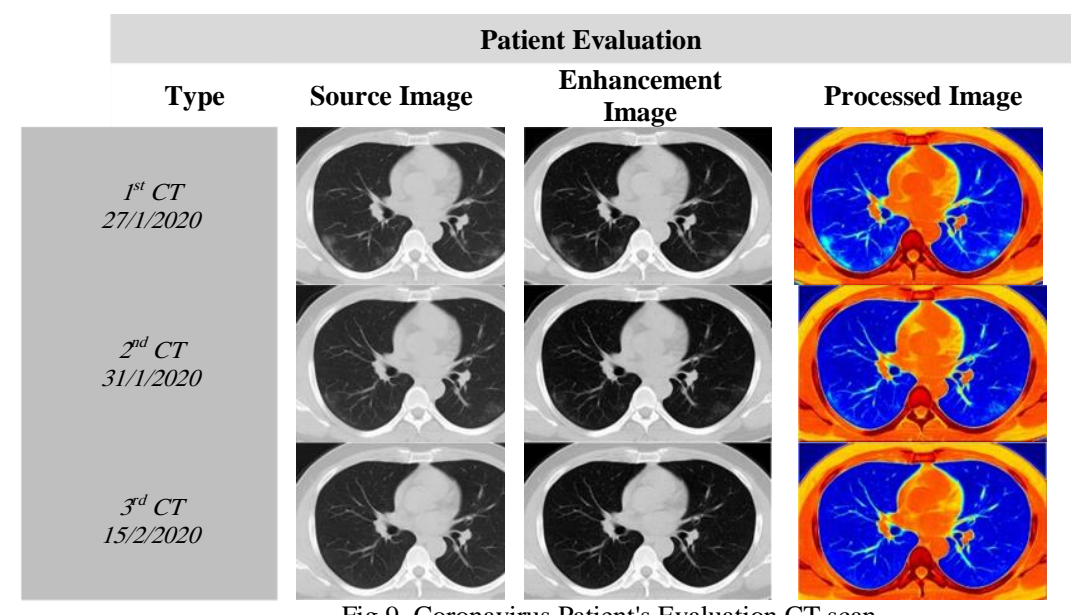

Fig.9. Coronavirus Patient's Evaluation CT scan 
TABLE 10

\begin{tabular}{|c|c|c|c|c|c|}
\hline \multicolumn{6}{|c|}{ COMPARATIVE EVALUATION } \\
\hline Method & Technique & Classification Objective & ACC. & SEN. & SPE. \\
\hline $\begin{array}{c}\text { C. Butt et. Al. } \\
\text { [26] }\end{array}$ & Deep learning & $\begin{array}{l}\text { COVID-19, Influenza viral } \\
\text { pneumonia, or no-infection }\end{array}$ & $98 \%$ & $98.2 \%$ & $92.2 \%$ \\
\hline L. Sun et al. [25] & AFS-DF & COVID-19, or no-infection & $91.8 \%$ & $93.1 \%$ & $89.9 \%$ \\
\hline Wang et al. [27] & $\mathrm{CNN}$ & $\begin{array}{l}\text { COVID-19, or typical viral } \\
\text { pneumonia }\end{array}$ & $73.1 \%$ & $67 \%$ & $74 \%$ \\
\hline Shi et al. [28] & Deep learning & $\begin{array}{c}\text { non-severe and severe } \\
\text { patients }\end{array}$ & $89 \%$ & $82.2 \%$ & $82.8 \%$ \\
\hline Tang et al [29] & $\begin{array}{l}\text { random forest } \\
\text { method }\end{array}$ & $\begin{array}{c}\text { severity of the COVID-19 } \\
\text { patient }\end{array}$ & $87.5 \%$ & $93.3 \%$ & $74.5 \%$ \\
\hline This Study & $\begin{array}{c}\text { Contrast } \\
\text { Enhancement and } \\
\text { Deep learning }\end{array}$ & COVD-19 CO-RADS & $98 \%$ & $\begin{array}{l}96.7- \\
100 \%\end{array}$ & $\begin{array}{l}97.5- \\
100 \%\end{array}$ \\
\hline
\end{tabular}

\section{CONCLUSION}

The Early detection of COVID-19 is an important and urgent need for stopping the spread of the disease. A developed deep learning classification technique was proposed using chest $\mathrm{CT}$ images. The objectives of the proposed technique are the classification and prediction of COVID-19 patients' grades automatically. As well as, the detection and the differentiation of coronavirus patients (CO-RADS 2 and CO-RADS 3) early grades for fast treatments. Deep transfer learning was done using ResNET50 pre-trained model. The technique accuracy is $98 \%$ for five grades classification. Several statistical parameters were obtained for different classification categories. The specificity and precision reach $100 \%$ in some classification categories. The minimum misclassification error is $1.67 \%$. These results can be easily enhancement by increasing the training datasets. The proposed technique will help physicians for faster decision making in clinical practice.

\section{REFERENCES}

[1] Z. Wu, J.M. McGoogan Characteristics of and important lessons from the coronavirus disease 2019 (COVID-19) outbreak in China: summary of a report of 72314 cases from the Chinese Center for Disease Control and Prevention, Jama, (2020), 10.1001/jama.2020.2648.

[2] Huang C, Wang Y, Li X, Ren L, Zhao J, Hu Y, et al. Clinical features of patients infected with 2019 novel coronavirus in Wuhan, China. Lancet DOI:https://doi.org/10.1016/S0140-6736(20)30183-5

[3] World Health Organization. Report of the WHO-China Joint Mission on Coronavirus Disease 2019 (COVID19). Geneva: WHO; 2020. Available from: https://www.who.int/docs/defaultsource/coronaviruse/whochina-joint-mission-on-covid-19-finalreport.pdf

[4] Bernheim A, Mei X, Huang M, Yang Y, Fayad ZA, Zhang N, Diao K, Zhu X, Li K, Li S, Shan H, Jacobi A, Chung M. Chest CT Findings in Coronavirus Disease-19 (COVID-10): Relationship to Duration of Infection. Radiology 2020. Doi: 10.1148/radiol.2020200463.

[5] Deng, J., Dong, W., Socher, R., Li, L.J., Li, K. and Fei-Fei, L.: Imagenet: A large-scale hierarchical image database. In: Computer Vision and Pattern Recognition (CVPR). [Online] Piscataway, New Jersey, USA: IEEE, pp.248-255. Available at: https://ieeexplore.ieee.org/document/5206848 [Accessed 18 Aug. 2009] DOI: $10.1109 /$ CVPR.2009.5206848
[6] Pan Y, Guan H, Zhou S, Wang Y, Li Q, Zhu T, Hu Q, Xia L. Initial $\mathrm{CT}$ findings and temporal changes in patients with the novel coronavirus pneumonia (2019-nCoV): a study of 63 patients in Wuhan, China. European radiology 2020. doi: 10.1007/s00330-02006731-x

[7] Kanne JP. Chest CT Findings in 2019 Novel Coronavirus (2019$\mathrm{nCoV}$ ) Infections from Wuhan, China: Key Points for the Radiologist. Radiology 2020:200241. doi: 10.1148/radiol.2020200241

[8] Heshui Shi XH, Nanchuan Jiang, Yukun Cao, Osamah Alwalid, Jin $\mathrm{Gu}$, Yanqing Fan, Chuansheng Zheng. Radiological findings from 81 patients with COVID-19 pneumonia in Wuhan, China: a descriptive study. The Lancet Infectious Diseases 2020. doi: https://doi.org/10.1016/S1473-3099(20)30086-4

[9] Center for Disease Control and Prevention. Interim Guidelines for Collecting, Handling, and Testing Clinical Specimens from Persons under Investigation (PUIs) for Coronavirus Disease 2019 (COVID19). $2020.2 \mathrm{https} / / / \mathrm{www} . c d c$. gov/coronavirus/2019ncov/lab/guidelines-clinical- specimens.html. Published February 14, 2020. Accessed March 5, 2020. Google Scholar.

[10] Fang Y, Zhang H, Xie J, Lin M, Ying L, Pang P, Ji W. Sensitivity of Chest CT for COVID19: Comparison to RT-PCR. Radiology 2020 Feb. 19:200432. DOI: 10.1148/radiol.2020200432

[11] Xie X, Zhong Z, Zhao W, Zheng C, Wang F, Liu, J. Chest CT for typical 2019-nCoV pneumonia: relationship to negative RT-PCR testing. Radiology 2020 Feb. 7. DOI: 10.1148/radiol.2020200343.

[12] Radiology Assistant Educational site of the Radiological Society of the Netherlands (COVID-19), Accessed April 1, 2020.

[13] H. Chen, J. Guo, C. Wang, F. Luo,et al ."Clinical characteristics and intrauterine vertical transmission potential of COVID-19 infection in nine pregnant women: a retrospective review of medical records". The Lancet, Volume 395, pp.809-815, DOI: 10.1016/S01406736(20)30360-3.

[14] P. van Kasteren, B. Veer, S. Brinka, L. Wijsman,et al ." Comparison of seven commercial RT-PCR diagnostic kits for COVID-19," Journal of Clinical Virology, Vol.128, 2020, https://doi.org/10.1016/j.jcv.2020.104412

[15] L. ZHONG, L. MU, J. LI, J. WANG, et al. "Early Prediction of the 2019 Novel Coronavirus Outbreak in the Mainland China Based on Simple Mathematical Model", IEEE Access, Volume 8, 2020, pp.51761-51769, DOI: 10.1109/ACCESS.2020.2979599.

[16] F. Rustam , A. Reshi , A. Mehmood , S. Ullah, et al." COVID-19 Future Forecasting Using Supervised Machine Learning Models," IEEE Access, 2020, Vol.8, pp.101489-101499, DOI: 10.1109/ACCESS.2020.2997311

[17] M. Alazab, A. Awajan, A. Mesleh, A. Abraham, et al ."COVID-19 Prediction and Detection Using Deep Learning," International Journal of Computer Information Systems and Industrial Management Applications. Vol.12, 2020, pp.168-181.

[18] Gozes, O., Frid-Adar, M., Greenspan, H., Browning, P. D., Zhang, H., Ji, W., Bernheim, A., and Siegel, E. Rapid AI Development Cycle for the Coronavirus (COVID-19) Pandemic: Initial Results for Automated Detection \& Patient Monitoring using Deep Learning CT Image Analysis. arXiv preprint arXiv:2003.05037, pp.1-19, 2020. 
[19] Shan, F., Gao, Y., Wang, J., Shi, W., Shi, N., Han, M., Xue, Z., and Shi, Y. Lung Infection Quantification of COVID-19 in CT Images with Deep Learning. arXiv preprint arXiv:2003.04655, 1-19, 2020.

[20] Xu, X., Jiang, X., Ma, C., Du, P., Li, X., Lv, S., Yu, L., Chen, Y., Su, J., Lang, G., Li, Y., Zhao, H., Xu, K., Ruan, L., and Wu, W. Deep Learning System to Screen Coronavirus Disease 2019 Pneumonia. arXiv preprint arXiv:2002.09334, pp.1-29, 2020.

[21] Wang, S., Kang, B., Ma, J., Zeng, X., Xiao, M., Guo, J., Cai, M., Yang, J., Li, Y., Meng,X., and Xu, B. A deep learning algorithm using CT images to screen for Corona Virus Disease (COVID-19). medRxiv preprint doi: https://doi.org/10.1101/2020.02.14.20023028, pp.1-26, 2020.

[22] H. Maghdid, A.Asaad, K.Ghafoor, A. Sadiq ,et al. "Diagnosing COVID-19 Pneumonia from X-Ray and CT Images using Deep Learning and Transfer Learning Algorithms, " arXiv preprint arXiv:2004.00038,2020, pp.1-8.

[23] X. Chen \& Y. Tang \& Y. Mo \& S. Li,"A diagnostic model for coronavirus disease 2019 (COVID-19) based on radiological semantic and clinical features: a multi-center study," European radiology, 2020, pp. 1-8, https://doi.org/10.1007/s00330-020-068292

[24] T. Ozturk, M. Talo, E. Yildirim, U. Baloglu, O. Yildirim, and U. Acharya, "Automated detection of COVID-19 cases using deep neural networks with X-ray images," Computers in Biology and Medicine, Elsevier, Vol.121, 2020, PP. 1-11, https://doi.org/10.1016/j.compbiomed.2020.103792

[25] L. Sun, Z. Mo, F. Yan, L. Xia, "Adaptive Feature Selection Guided Deep Forest for COVID-19 Classification with Chest CT," arXiv preprint arXiv: 2005.03264, 2020.

[26] C. Butt, J. Gill, D. Chun, and B. A. Babu, "Deep learning system to screen coronavirus disease 2019 pneumonia", Nature Public Health Emergency Collection, 2020, pp: 1-1, doi: 10.1007/s10489-02001714-3
[27] S. Wang, B. Kang, J. Ma, X. Zeng, M. Xiao, J. Guo, M. Cai, J. Yang, Y. Li, X. Meng, et al., "A deep learning algorithm using CT images to screen for Corona Virus Disease (covid-19)," medRxiv, 2020.

[28] W. Shi, X. Peng, T. Liu, Z. Cheng, et al., "Deep learning-based quantitative computed tomography model in predicting the severity of COVID-19: A retrospective study in 196 patients," 2020

[29] Z. Tang, W. Zhao, X. Xie, Z. Zhong, F. Shi, J. Liu, and D. Shen, "Severity assessment of coronavirus disease 2019 (COVID-19) using quantitative features from chest CT images," arXiv preprint arXiv: 2003.11988, 2020.

\section{Arabic Title}

طريقة آليـة للاكتثـاف المبكر و التصنيف لمر احل فيروس كورونـا )

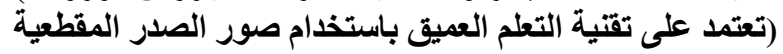

\section{Arabia Abstract}

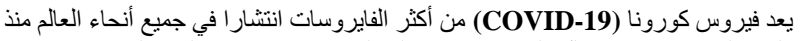

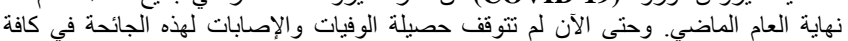

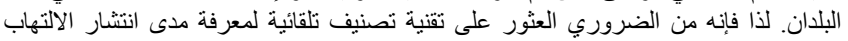

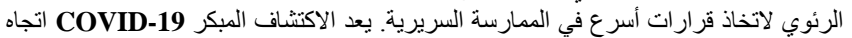

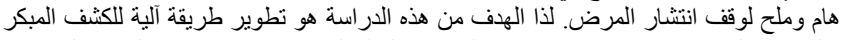

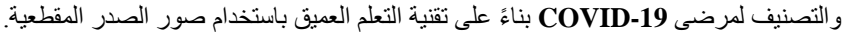

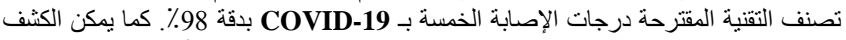
عن درجات الإصابة المبكرة بدقة 98.3\%. تم تقيبم العديد من مقاييس الأداء الإحصائي لفئات

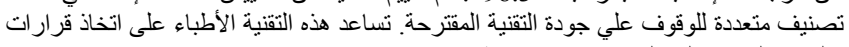
و العلاج المناسب لمختلف درجات الاصنابة.COVID-19 